\title{
Prognostic Value of the Six-Minute Walk Test in Heart Failure Patients Undergoing Cardiac Surgery: A Literature Review
}

\author{
Dominika Zielińska, ${ }^{1}$ Jerzy Bellwon, ${ }^{2}$ Andrzej Rynkiewicz, ${ }^{2}$ and Mohamed Amr Elkady ${ }^{3}$ \\ ${ }^{1}$ Clinic of Rehabilitation, Medical University of Gdańsk, ul. Dębinki 7, 80-952 Gdańsk, Poland \\ ${ }^{2}$ Department of Cardiology, Medical University of Gdansk, Poland \\ ${ }^{3}$ Department of Physical Medicine \& Rehabilitation, Ain Shams University, Cairo, Egypt
}

Correspondence should be addressed to Dominika Zielińska; dziel@gumed.edu.pl

Received 3 April 2013; Accepted 3 July 2013

Academic Editor: Richard Crevenna

Copyright (C) 2013 Dominika Zielińska et al. This is an open access article distributed under the Creative Commons Attribution License, which permits unrestricted use, distribution, and reproduction in any medium, provided the original work is properly cited.

\begin{abstract}
Background. The prognostic value of cardiopulmonary exercise testing (CPET) is known, but the predictive value of 6MWT in patients with heart failure (HF) and patients undergoing coronary artery bypass grafting (CABG) is not established yet. Objective. We conducted a systematic review exploring the prognostic value of 6MWT in HF patients undergoing cardiac surgery. The aim was to find out whether the change in the distance walked during follow-up visits was associated with prognosis. Data Source. We searched "PubMed" from January 1990 to December 2012 for any review articles or experimental studies investigating the prognostic value of 6MWT in HF patients and patients undergoing cardiac surgery. Results. 53 studies were included in the review, and they explored the role of 6MWT in cardiology, cardiac surgery, and rehabilitation. The results did not show the relation between the six-minute walk distance and adverse events after CABG. The predictive power of the distance walked for death in HF patients undergoing cardiac surgery was not found. It is not yet proved if the change in the six-minute walk distance is associated with prognosis. The predictive power of the six-minute walk distance for death in HF patients undergoing cardiac surgery remains unclear.
\end{abstract}

\section{Background}

1.1. Definition and History of Implementation of the Six-Minute Walk Test. The six-minute walk test is a simple, inexpensive, and reproducible method for the assessment of exercise capacity. Implementation of the test does not require any advanced equipment or training for technicians. During the test, the patient walks the longest possible distance within the time of 6 minutes on the flat surface and can stop or slow down at any time and then resume walking during the test. The main result of the six-minute walk test (6MWT) is the distance covered by the patient in 6 minutes. The $6 \mathrm{MWT}$ shows good correlation with the peak VO2 from cardiopulmonary exercise test and is much easier to perform, and it reflects well the daily activities of the patients. However, many variables may influence this test, and, therefore, it should always be performed according to the strict given protocol. The 6MWT was proposed for the first time by Balke in 1963, and since the mid-1980s, it has been used more and more widely in different clinical conditions. This test is most commonly used in pulmonary diseases, but it has been successfully implemented also in patients with cardiovascular diseases, pre- and postsurgical treatment, different neurological disorders, and fibromyalgia or spinal muscular atrophy. However, it seems that the 6MWT is not so popular among cardiologists and cardiosurgeons as it should be.

1.2. Six-Minute Walk Test as a Tool for Assessment of Clinical Condition and Prognosis in Cardiovascular Diseases. Exercise capacity and tolerance are the most important factors in assessment of the clinical condition and prognosis of patients with cardiovascular and pulmonary diseases. Exercise capacity is the strong prognostic factor in heart failure patients and can be best described by cardiopulmonary exercise testing (CPET), mainly measuring the peak oxygen consumption. 
Unfortunately, such test requires complicated and expensive equipment, qualified technicians, experienced physicians, and frequent gas and volume calibration. All that makes CPET a complicated method reserved for specialized facilities.

Several different walk tests have been described in effort to measure the functional capacity, including 2-minute walk test (2MWT), 6MWT, 12MWT, self-paced walk test, shuttle walk test, and 1-mile track walk. All these tests are inexpensive and relatively simple to perform. Among them, the 6MWT seems to be the most frequently used for clinical and research purposes. This test was proposed for the first time in cardiology by Balke [1] in 1963 and then by Guyatt et al. [2] in heart failure patients. The 6MWT distance (6MWTD) seems to correlate best with the maximal oxygen consumption. This test is easy to be administrated, inexpensive, well tolerated by patients, and reflects their daily activities.

The 6MWT does not measure the peak oxygen uptake or determine the cause of dyspnea on exertion, but it correlates well $(r=0,73)$ with VO2 peak in some patients with endstage lung diseases.

Anyway, the 6MWT distance correlates better with the quality of life indices than with VO2 peak, and this demonstrates the 6MWT better suitability for assessing patients' ability in performing their daily activities than the CPX test [3].

In fact, there are many protocols of the 6MWT but the differences between them are usually small, but the most detailed and widely implemented protocol was published by the American Thoracic Society in 2002 [3].

The assessment of exercise capacity by means of the $6 \mathrm{MWT}$ is most frequently used in pulmonary and cardiac diseases. This test measures the distance a person can quickly walk on a flat, hard surface in the time of 6 minutes (the $6 \mathrm{MWD})$. The $6 \mathrm{MWT}$ requires a 30 -meter $(100 \mathrm{ft})$ corridor, stopwatch, mechanical lap counter, two small cones to mark the turnover points, one chair that can be easily moved along the walking course to support the patient, worksheets on a clipboard, an available source of oxygen, a sphygmomanometer or other validated blood pressure measuring devices, a telephone, and a defibrillator. The length of the hallway should be marked every 3 meters with a cone, and the starting line should be marked on the floor using brightly colored tape. In case of repeating the test, it is important that it should be performed at the same time of the day and without any "warmup." The patient should rest seated on a chair located near the starting line for at least 10 minutes before the test starts. Meanwhile, the contraindications for the test should be checked and identified; the pulse and blood pressure should be measured. Performing pulse oximetry is optional. The baseline and overall fatigue should be assessed using the Borg scale [4]. Before starting the test, the patient is instructed to walk as far as possible for 6 minutes, and during the walk test, only standardized phrases for encouragement must be used. After the test, the postwalk dyspnea and fatigue should be assessed by Borg scale, as well as the pulse and blood pressure. If the oximeter was used, the Sat $\mathrm{O} 2$ should be recorded, right after the test. The number of laps from the counter should be recorded and additional distance covered should be also marked with the use of the markers on the wall as distance guides to calculate the total distance of walk.

The normative data of the 6MWTD are based on several studies performed on healthy populations, and their results serve as a reference point for better understanding and proper interpretation of the $6 \mathrm{MWT}$ results. The 6MWT distance depends on anthropometric variables like age, gender, and weight. It also depends on the protocol specifications, mainly on verbal encouragement for the patient to continue the test and also on the results obtained by the patient in learning how to perform the test [5].

In the study of Gibbons et al. [6] in which younger people whose mean age was 45.1 years were examined and included, the participants achieved a mean 6MWT distance of 689 meters for men and 615 meters for women.

Among other studies of more elderly people, Troosters et al. [7] reported a mean $6 \mathrm{MWT}$ distance of 613 meters in subjects whose mean age was 65 years, and the results reported were 656 meters for men and 554 meters for women.

Steffen et al. reported a mean 6MWT distance of 505 meters for men and of 467 meters for women aged 74.1 on average [8].

Another study performed by Enrichi and Sherrill [9] provided normative data in healthy adults. They have examined 117 healthy men and 173 healthy women aged from 40 to 80 years, and the mean 6MWT distance was 576 meters for men and 494 meters for women. Based on those data, they developed equations allowing adjustment for gender, age, height, and weight to calculate the distance walked by a healthy adult during the 6MWT.

1.3. Six-Minute Walk Distance as a Prognostic Factor in Heart Failure Patients. Several studies have reported that the $6 \mathrm{MWT}$ is a reliable measure of increased mortality among cardiac patients, with the distance of less than 300 meters being a strong indicator of poor prognosis [10]. The 6MWT distance in patients with heart failure and left ventricular ejection fraction (LVEF) of 20\% averages at 310 meters [11], whereas in those with mild disease and preserved LVEF $(>53 \%)$, it is over 427 meters [12].

In patients with heart failure, a low 6-minute walk distance has been associated with increased total mortality and more hospital admissions for heart failure [13, 14]. The 6-minute walk distance is only weakly correlated with LV ejection fraction, and it provides independent prognostic information $[13,14]$.

The 6MWT is a safe and simple clinical method; that is, it strongly and independently allows us to predict heart failure hospitalization rates and mortality in patients with left ventricular dysfunction. The mortality was 3.5 times higher in subjects covering less than 350 meters in the 6MWT than in those who walked over 450 meters in the Studies of Left Ventricular Dysfunction (SOLVD) registry substudy [15]. Other studies support the usefulness of the 6MWT distance in predicting not only mortality but also hospitalization for heart failure $[13,16]$.

The 6MWT can differentiate the most severe heart failure patients from the ones with mild to moderate diseases. 
The 6MWD is inversely related to New York Heart Association (NYHA) functional class and quality of life (QoL). However, only the physical functioning sections of healthrelated quality of life questionnaires, like SF-36 or MLHFQ, correlate significantly with the $6 \mathrm{MWT}$ distance $[17,18]$. In contrast, the nonphysical domains of quality of life do not correlate with the 6MWT.

Peak VO2 is a strong indicator of heart failure severity and is an important factor in timing of heart transplantation, and the 6MWT distance is strongly correlated with peak $\mathrm{VO} 2$ in HF patients with reported correlation coefficient in the range from $r=0.56$ to $r=0.88$ [5]. The correlation between $6 \mathrm{MWT}$ and peak VO2 in patients with heart failure is stronger in patients with low 6MWT and low peak VO2; then, 6MWT becomes less predictive as peak VO2 value becomes higher. The $6 \mathrm{MWT}$ is reliable, valid, and predictive for patients with heart failure who do not walk greater than 490 meters.

However, others have not confirmed this relationship, and they suggest that VO2 peak is a better predictor of survival, particularly over longer followup periods [19-22].

Some authors suggest that a submaximal exercise test could reflect the results obtained from a maximal exercise test in people whose physical functional capacity is severely impaired. However, maximal exercise testing may be more precise in those with severe heart failure who are referred for heart transplantation [3].

There are somehow conflicting results on using the $6 \mathrm{MW}$ distance as a marker of improvement following medical therapy for heart failure. In some cases, treatment with betablockers, angiotensin II blockers, or ACE inhibiters in general did not increase the 6MW distance despite the increase in LVEF and NYHA functional class [23-25].

An analysis by Olsson et al. [26] summarizing 39 studies related to the usefulness of the $6 \mathrm{MWT}$ as a measure of the medical treatment effectiveness presented only nine significant results. The majority of the pharmacological trials did not show any significant changes in the 6MWT distance. Nevertheless, a mean of 41 meters decrease in the $6 \mathrm{MW}$ distance has been observed by Packer et al. after digoxin withdrawal in patients with LVEF 35\% in the RADIANCE Study [27]. Changes in 6MWT distance correlate better with changes in symptoms, and it may be used as supportive evidence for symptom benefit following different means of treatment in heart failure patients. Similarly, small but significant improvements in the $6 \mathrm{MW}$ distance have been documented by several studies after cardiac resynchronization device implantation [28-30] and during the treatment with continuous positive airway pressure [31].

The test seems to be also safe in patients with refractory heart failure during the preoperative period for heart transplant surgery [32]. Clearer and more robust documentation is available on improvement in functional exercise capacity measured by the 6MWT after cardiac rehabilitation [3335]. The $6 \mathrm{MWT}$ is considered safe to assess the submaximal functional capacity and can be used as an alternative test to evaluate the results of interventions in patients with permanent cardiac pacemakers [36].
Absolute contraindications for the test include a history of unstable angina or a heart attack during the previous month. Relative contraindications are resting tachycardia HR $>120$ beats/min or uncontrolled arterial hypertension [37].

The test strongly and independently predicts morbidity and mortality in patients with heart failure, and it is more sensitive to deterioration than to improvement in heart failure symptoms [15].

\subsection{Six-Minute Walk Test in Patients Undergoing Cardiac} Surgery. Another field of application of walk test is postsurgical cardiac and pulmonary rehabilitation. In the literature, we can find reference values of the 6MWT in patients early after cardiac surgery [37, 38]. Some authors used the test in patients after lung volume reduction surgery in order to compare median sternotomy and thoracoscopic approaches.

Slow walking speed is a component of frailty and is associated with poor muscle strength and reduced mobility. In recent studies, frailty was a significant independent predictor of mortality or need for institutional care after cardiac surgery [39]. In 133 elderly patients undergoing cardiac surgery, Afilalo et al. reported that slow gait speed defined as the time taken to walk 5 meters in $>6$ seconds was associated with a higher risk of in-hospital complications from surgery based on the STS criteria. This small study did not report associations with long-term outcomes [40, 41]. In patients referred for surgery for severe aortic stenosis, the six-minute walk distance predicted death, myocardial infarction, and stroke events independently from the EuroSCORE [42].

\section{Conclusion}

The relation between the six-minute walk distance and adverse events after CABG has not been evaluated. The predictive power of the six-minute walk distance for death in heart failure patients undergoing cardiac surgery was not assessed as well. The question: "is change in the six-minute walk distance during follow-up visits associated with prognosis in heart failure patients?" also remains unanswered.

\section{References}

[1] B. Balke, "A simple field test for the assessment of physical fitness," Report Civil Aeromedical Research Institute (U.S.), vol. 53, pp. 1-8, 1963.

[2] G. H. Guyatt, M. J. Sullivan, P. J. Thompson et al., "The 6minute walk: a new measure of exercise capacity in patients with chronic heart failure," Canadian Medical Association Journal, vol. 132, no. 8, pp. 919-921, 1985.

[3] ATS Committee on Proficiency Standards for Clinical Pulmonary Function Laboratories, "ATS Statement: guidelines for the six-minute walk test," The American Journal of Respiratory and Critical Care Medicine, vol. 166, no. 1, pp. 111-117, 2002.

[4] G. A. V. Borg, "Psycho-physical bases of perceived exertion," Medicine and Science in Sports and Exercise, vol. 14, pp. 377-381, 1982.

[5] H. Du, P. J. Newton, Y. Salamonson, V. L. Carrieri-Kohlman, and P. M. Davidson, "A review of the six-minute walk test: Its 
implication as a self-administered assessment tool," European Journal of Cardiovascular Nursing, vol. 8, no. 1, pp. 2-8, 2009.

[6] W. J. Gibbons, N. Fruchter, S. Sloan, and R. D. Levy, "Reference values for a multiple repetition 6-minute walk test in healthy adults older than 20 years," Journal of Cardiopulmonary Rehabilitation, vol. 21, no. 2, pp. 87-93, 2001.

[7] T. Troosters, R. Gosselink, and M. Decramer, "Six minute walking distance in healthy elderly subjects," European Respiratory Journal, vol. 14, no. 2, pp. 270-274, 1999.

[8] T. M. Steffen, T. A. Hacker, and L. Mollinger, "Age- and genderrelated test performance in community-dwelling elderly people: six-minute walk test, Berg balance scale, timed up \& go test, and gait speeds," Physical Therapy, vol. 82, no. 2, pp. 128-137, 2002.

[9] P. L. Enrichi and D. L. Sherrill, "Reference equations for the six-minute walk in healthy adults," The American Journal of Respiratory and Critical Care Medicine, vol. 158, no. 5, part 1, pp. 1384-1387, 1998.

[10] I. Daullxhiu, E. Haliti, A. Poniku et al., "Predictors of exercise capacity in patients with chronic heart failure," Journal of Cardiovascular Medicine, vol. 12, no. 3, pp. 223-225, 2011.

[11] L. P. Cahalin, M. A. Mathier, M. J. Semigran, G. W. Dec, and T. G. DiSalvo, "The six-minute walk test predicts peak oxygen uptake and survival in patients with advanced heart failure," Chest, vol. 110, no. 2, pp. 325-332, 1996.

[12] C. Rostagno, G. Olivo, M. Comeglio et al., "Prognostic value of 6-minute walk corridor test in patients with mild to moderate heart failure: comparison with other methods of functional evaluation," European Journal of Heart Failure, vol. 5, no. 3, pp. 247-252, 2003.

[13] V. Bittner, "Determining prognosis in congestive heart failure: role of the 6-minute walk test," The American Heart Journal, vol. 138, no. 4, pp. 593-596, 1999.

[14] L. Ingle, A. S. Rigby, S. Carroll et al., "Prognostic value of the 6 min walk test and self-perceived symptom severity in older patients with chronic heart failure," European Heart Journal, vol. 28, no. 5, pp. 560-568, 2007.

[15] V. Bittner, D. H. Weiner, S. Yusuf et al., "Prediction of mortality and morbidity with a 6-minute walk test in patients with left ventricular dysfunction," The Journal of the American Medical Association, vol. 270, no. 14, pp. 1702-1707, 1993.

[16] R. Boxer, A. Kleppinger, A. Ahmad, K. Annis, D. Hager, and A. Kenny, "The 6-minute walk is associated with frailty and predicts mortality in older adults with heart failure," Congestive Heart Failure, vol. 16, no. 5, pp. 208-213, 2010.

[17] S. R. Lord and H. B. Menz, "Physiologic, psychologic, and health predictors of 6-minute walk performance in older people," Archives of Physical Medicine and Rehabilitation, vol. 83, no. 7, pp. 907-911, 2002.

[18] C. Demers, R. S. McKelvie, A. Negassa, and S. Yusuf, "Reliability, validity, and responsiveness of the six-minute walk test in patients with heart failure," The American Heart Journal, vol. 142, no. 4, pp. 698-703, 2001.

[19] C. Zugck, C. Krüger, S. Dürr et al., "Is the 6-minute walk test a reliable substitute for peak oxygen uptake in patients with dilated cardiomyopathy?" European Heart Journal, vol. 21, no. 7, pp. 540-549, 2000.

[20] J. G. Lainchbury and A. M. Richards, "Exercise testing in the assessment of chronic congestive heart failure," Heart, vol. 88, no. 5, pp. 538-543, 2002.
[21] C. Pulz, R. V. Diniz, A. N. F. Alves et al., "Incremental shuttle and six-minute walking tests in the assessment of functional capacity in chronic heart failure," Canadian Journal of Cardiology, vol. 24, no. 2, pp. 131-135, 2008.

[22] D. J. Lederer, S. M. Arcasoy, J. S. Wilt, F. D’Ovidio, J. R. Sonett, and S. M. Kawut, "Six-minute-walk distance predicts waiting list survival in idiopathic pulmonary fibrosis," The American Journal of Respiratory and Critical Care Medicine, vol. 174, no. 6, pp. 659-664, 2006.

[23] M. Packer, W. S. Colucci, J. D. Sackner-Bernstein et al., "Doubleblind, placebo-controlled study of the effects of carvedilol in patients with moderate to severe heart failure. The PRECISE Trial. Prospective randomized evaluation of carvedilol on symptoms and exercise," Circulation, vol. 94, no. 11, pp. 27932799, 1996.

[24] Australia/New Zealand Heart Failure Research Collaborative Group, "Randomised, placebo-controlled trial of carvedilol in patients with congestive heart failure due to ischaemic heart disease," The Lancet, vol. 349, pp. 375-380, 1997.

[25] R. S. McKelvie, S. Yusuf, D. Pericak et al., "Comparison of candesartan, enalapril, and their combination in congestive heart failure: randomized evaluation of strategies for left ventricular dysfunction (RESOLVD) pilot study: the RESOLVD pilot study investigators," Circulation, vol. 100, no. 10, pp. 1056-1064, 1999.

[26] L. G. Olsson, K. Swedberg, A. L. Clark, K. K. Witte, and J. G. F. Cleland, "Six minute corridor walk test as an outcome measure for the assessment of treatment in randomized, blinded intervention trials of chronic heart failure: a systematic review," European Heart Journal, vol. 26, no. 8, pp. 778-793, 2005.

[27] M. Packer, M. Gheorghiade, J. B. Young et al., "Withdrawal of digoxin from patients with chronic heart failure treated with angiotensin-converting-enzyme inhibitors," The New England Journal of Medicine, vol. 329, no. 1, pp. 1-7, 1993.

[28] W. T. Abraham, W. G. Fisher, A. L. Smith et al., "Cardiac resynchronization in chronic heart failure," The New England Journal of Medicine, vol. 346, no. 24, pp. 1845-1853, 2002.

[29] S. Agosti, L. Casalino, G. Bertero et al., "Selection of candidates for cardiac resynchronisation therapy and prediction of their response," Clinica Terapeutica, vol. 161, no. 2, pp. 143-148, 2010.

[30] A. Achilli, M. Sassara, D. Pontillo et al., "Effectiveness of cardiac resynchronisation therapy in patients with echocardiographic evidence of mechanical dyssynchrony," Journal of Cardiovascular Medicine, vol. 9, no. 2, pp. 131-136, 2008.

[31] T. D. Bradley, A. G. Logan, R. J. Kimoff et al., "Continuous positive airway pressure for central sleep apnea and heart failure," The New England Journal of Medicine, vol. 353, no. 19, pp. 2025-2033, 2005.

[32] G. Cipriano Jr., G. F. Bernardelli, R. Arena, L. V. F. Oliveira, F. Valdez, and J. N. R. Branco, "Cardio-respiratory responses of the 6-minute walk test in patients with refractory heart failure during the preoperative period for heart transplant surgery," Monaldi Archives for Chest Disease_Cardiac Series, vol. 74, no. 2, pp. 64-69, 2010.

[33] V. Gremeaux, O. Troisgros, S. Benam et al., "Determining the minimal clinically important difference for the six-minute walk test and the 200-meter fast-walk test during cardiac rehabilitation program in coronary artery disease patients after acute coronary syndrome," Archives of Physical Medicine and Rehabilitation, vol. 92, no. 4, pp. 611-619, 2011.

[34] K. Rees, R. S. Taylor, S. Singh, A. J. Coats, and S. Ebrahim, "Exercise based rehabilitation for heart failure," Cochrane Database of Systematic Reviews, no. 3, Article ID CD003331, 2004. 
[35] V. Gremeaux, G. Deley, J. Duclay, D. Antoine, A. Hannequin, and J. M. Casillas, "The 200-m fast-walk test compared with the 6-min walk test and the maximal cardiopulmonary test: a pilot study," The American Journal of Physical Medicine and Rehabilitation, vol. 88, no. 7, pp. 571-578, 2009.

[36] L. A. P. de Sousa, R. R. Britto, A. L. Ribeiro et al., "Six-minute walk test in patients with permanent cardiac pacemakers," Journal of Cardiopulmonary Rehabilitation and Prevention, vol. 28, no. 4, pp. 253-257, 2008.

[37] C. Fiorina, E. Vizzardi, R. Lorusso et al., “The 6-min walking test early after cardiac surgery. Reference values and the effects of rehabilitation programme," European Journal of Cardio-thoracic Surgery, vol. 32, no. 5, pp. 724-729, 2007.

[38] O. Bettinardi, G. Bertolotti, P. Baiardi et al., "Can influence anxiety and depression the six-minute walking test performance in post-surgical heart valve patients? A pilot study," Monaldi Archives for Chest Disease-Cardiac Series, vol. 62, no. 3, pp. 154-161, 2004.

[39] D. H. Lee, K. J. Buth, B. Martin, A. M. Yip, and G. M. Hirsch, "Frail patients are at increased risk for mortality and prolonged institutional care after cardiac surgery," Circulation, vol. 121, no. 8, pp. 973-978, 2010.

[40] J. Afilalo, M. J. Eisenberg, J. Morin et al., "Gait speed as an incremental predictor of mortality and major morbidity in elderly patients undergoing cardiac surgery," Journal of the American College of Cardiology, vol. 56, no. 20, pp. 1668-1676, 2010.

[41] S. Sündermann, A. Dademasch, A. Rastan et al., "One-year follow-up of patients undergoing elective cardiac surgery assessed with the comprehensive assessment of frailty test and its simplified form," Interactive Cardiovascular and Thoracic Surgery, vol. 13, no. 2, pp. 119-123, 2011.

[42] D. P. de Arenaza, J. Pepper, B. Lees et al., "Preoperative 6-minute walk test adds prognostic information to Euroscore in patients undergoing aortic valve replacement," Heart, vol. 96, no. 2, pp. 113-117, 2010. 


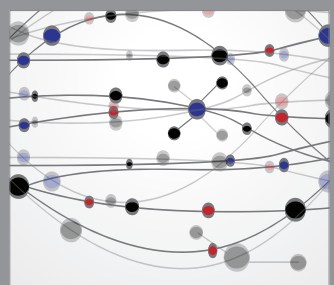

The Scientific World Journal
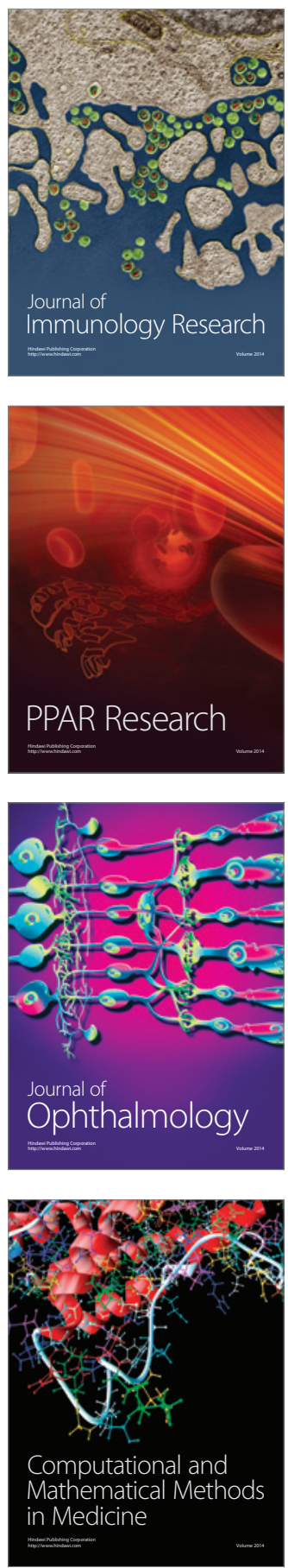

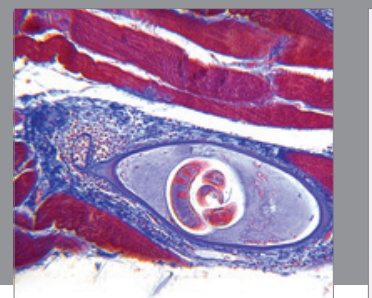

Gastroenterology

Research and Practice
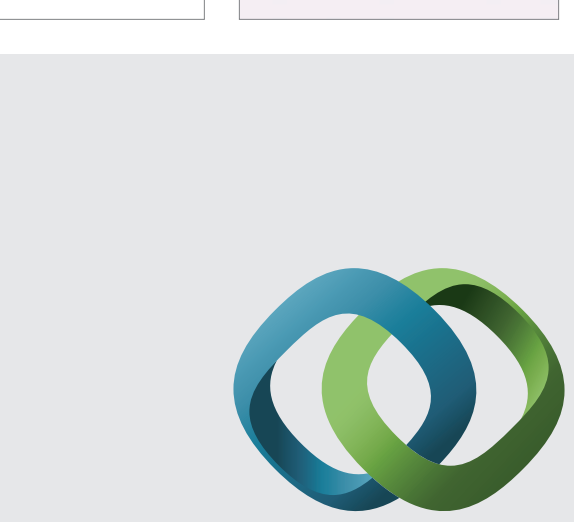

\section{Hindawi}

Submit your manuscripts at

http://www.hindawi.com
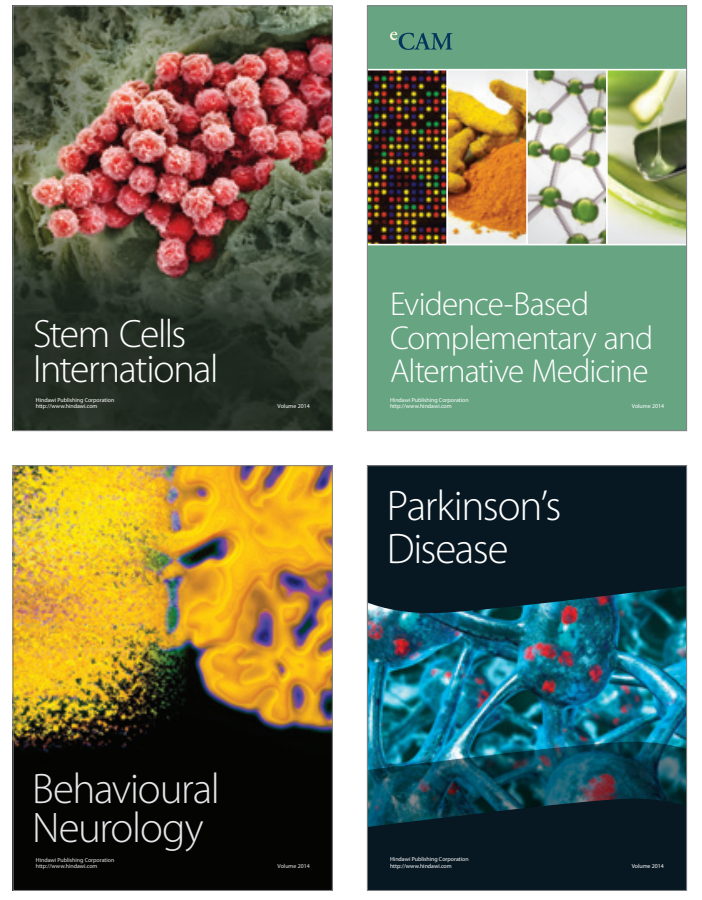
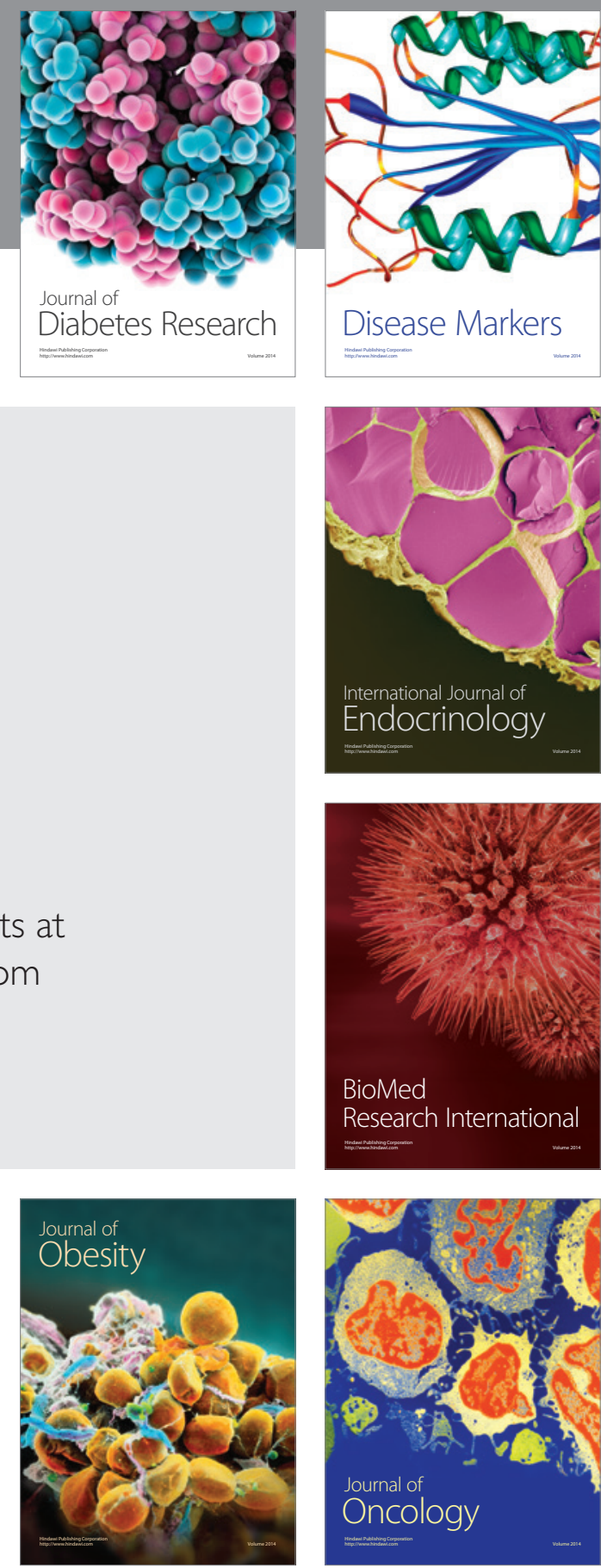

Disease Markers
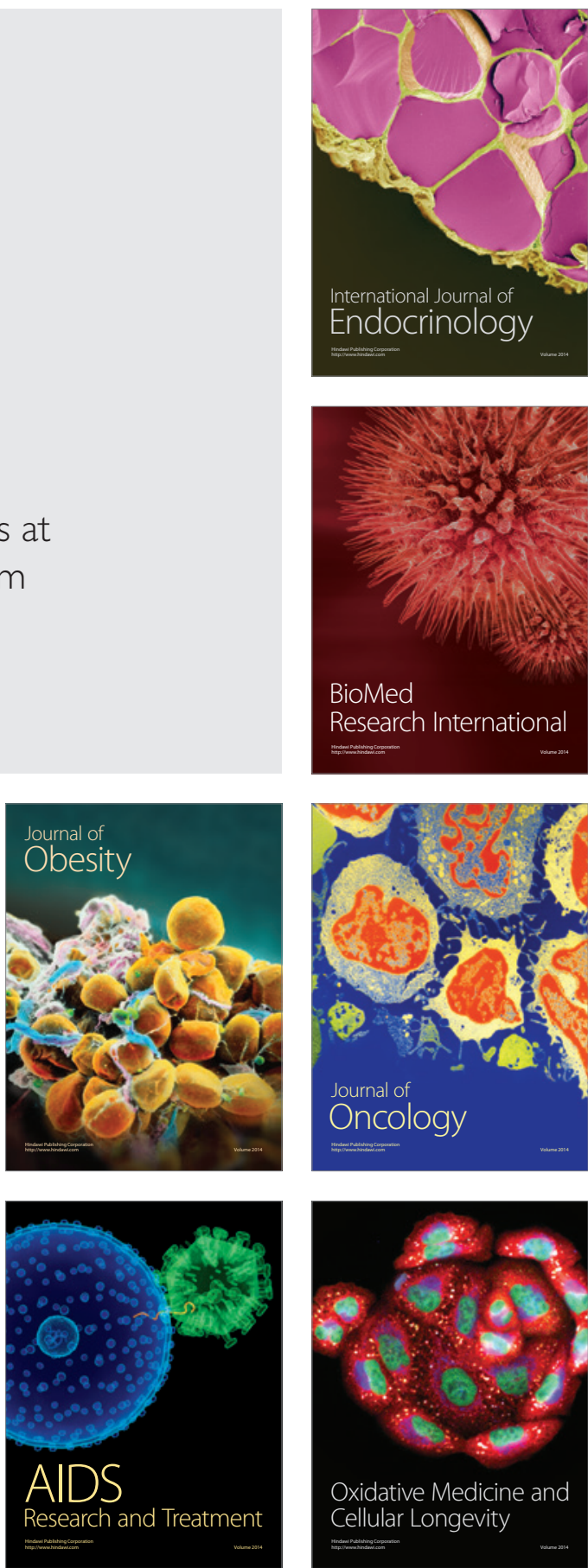